\title{
Process R\&D investment and social dilemmas
}

\author{
Michal Ramsza ${ }^{1} \cdot$ Adam Karbowski $^{2}$ (D) Tadeusz Platkowski ${ }^{3}$
}

Received: 11 August 2020 / Revised: 12 April 2021 / Accepted: 16 April 2021 / Published online: 26 April 2021

(c) The Author(s) 2021

\begin{abstract}
We consider a coopetitive game model of firms' behavior in process R\&D with entry cost. We compare the competitive behavior of firms in $R \& D$ with the $R \& D$ coopetition scenario. In $R \& D$ coopetition, firms engage in a bargaining process to reach a binding $R \& D$ agreement. We find that $R \& D$ competition can lead to a prisoner's dilemma or a chicken game between market rivals. The possibility of entering a binding $R \& D$ agreement resolves the above social dilemmas associated with the firms' competitive behavior. In turn, under R\&D coopetition, for a medium level of $\mathrm{R} \& \mathrm{D}$ entry cost, firms may enter a trust dilemma, but it is a beneficial scenario in comparison with the corresponding R\&D competition outcome.
\end{abstract}

Keywords R\&D investment · Bargaining $\cdot$ Competition · Coopetition · Social dilemmas

JEL Classification D02 - D21 · O3

Adam Karbowski

akarbo1@sgh.waw.pl

Michal Ramsza

michal.ramsza@gmail.com

Tadeusz Platkowski

tplatk@mimuw.edu.pl

1 Department of Mathematics and Mathematical Economics, SGH Warsaw School of Economics, Al. Niepodleglos̀ci 162, 02-554 Warsaw, Poland

2 Department of Business Economics, Collegium of World Economy, SGH Warsaw School of Economics, Al. Niepodleglos̀ci 162, 02-554 Warsaw, Poland

3 Faculty of Mathematics, Informatics and Mechanics, University of Warsaw, ul. Banacha 2, 02-097 Warsaw, Poland 


\section{Introduction}

$R \& D$ coopetition (cooperation in $R \& D$ between market rivals) is used in various industries, cf., e.g., Bouncken and Fredrich (2016), Cygler et al. (2018), Jakobsen (2020), to achieve technological synergies and cost reductions, among other benefits (see, e.g., Ritala and Sainio (2014) or Conti and Marini (2019)). Interestingly, R\&D coopetition can also extricate firms from disadvantageous social dilemmas, as we further show in this article. The present paper contributes to the relevant innovation literature by considering $R \& D$ behavior of firms from various social dilemma viewpoints (prisoner's dilemma, chicken game, and trust dilemma). The particularly interesting contribution of our article extends the well-known finding discussed by Amir et al. (2011). The latter authors show that under spillover levels not too high and relatively low R\&D costs, Cournot firms are caught in the prisoner's dilemma for their R\&D investment decisions. As we show, this result can be developed when the $R \& D$ fixed entry costs are introduced into the analysis. To be specific, if the entry costs are greater than 70 per cent and smaller than 100 per cent of the initial marginal costs, our results qualitatively differ and extend the findings discussed by Amir et al. (2011).

As we noted, economists have already investigated the R\&D behavior of firms from the social dilemma perspective. However, in the previous works, this perspective was used in a rather limited way, sometimes only as a by-product of a standard economic analysis. We set out to exploit the social dilemma perspective in firms' $R \& D$ in a broader way compared with the relevant innovation literature reviewed below. Lambertini and Rossini (1998) showed that firms may compete in undifferentiated products due to a prisoner's dilemma generated by externalities affecting R\&D in product innovation. Amir et al. (2011) considered a standard duopoly two-stage game of process R\&D and quantity competition. They showed that competing firms are caught in a prisoner's dilemma for their R\&D decisions whenever technological spillovers in the industry are low and costs of conducting R\&D are not too high. Such a prisoner's dilemma underlies the creation of an R\&D-avoiding cartel. Burr et al. (2013) extended the result that duopoly firms end up in a prisoner's dilemma for their R\&D decisions, whenever technological spillovers and R\&D costs are relatively low. In particular, they showed that incentives faced towards $R \& D$ cartel are maximal for the case of zero spillovers, which is when the prisoner's dilemma has the largest scope.

In the present paper, we identify not only prisoner's dilemma, but also two other fundamental social dilemmas — chicken game and trust dilemma-in firms' R\&D investment decisions. We further show that in each of the distinct dilemmas, a possibility to enter a binding $R \& D$ agreement and start $R \& D$ coopetition changes a competitive outcome to a more desirable one. In general, we show that disadvantageous social dilemmas associated with the firms' competitive behavior are mitigated by $R \& D$ coopetition. The latter result is in line with the relevant innovation literature, where the beneficial role of $R \& D$ agreements has been already identified. For example, Conti and Marini (2019) show that interfirm 
R\&D agreements can effectively enhance enterprise gains from the internalization of industrial knowledge spillovers.

Since social dilemmas are crucial to our paper, let us briefly differentiate between the basic types of social dilemmas. In the prisoner's dilemma game, players face two social incentives, i.e., the gain for those who exploit cooperative partners (greed), and the loss for cooperators who are exploited by non-cooperative partners (fear), see (Płatkowski 2017). A near-cousin of the prisoner's dilemma game, trust dilemma (assurance game), cf. (Kiyonari et al. 2000), is characterized by a different social tension than prisoner's dilemma. In the trust dilemma only fear is present. Finally, a chicken game is a social dilemma in which only greed is present.

We introduce the social dilemma perspective into the broader literature on strategic behavior of firms in R\&D which in turn is a straightforward continuation of the debate initiated by Schumpeter (1942) on the relationship between industry structure and incentives to undertake R\&D. In the relevant following literature, cf., e.g., Spence (1984), Katz (1986), d'Aspremont and Jacquemin (1988), Kamien et al. (1992), Kamien and Zang (2000), Amir et al. (2011), Burr et al. (2013), Bourreau et al. (2016), Capuano and Grassi (2019), the behavior of firms in R\&D is modeled by non-cooperative games (see also Cosandier et al. 2017 or Amir et al. 2019), in which enterprises, first, simultaneously and independently decide about their R\&D investments (these decisions affect the total manufacturing costs of each enterprise), and, further, compete in the final product market according to a given (quantity or price) competition model. To be specific, our paper is directly related to works by Amir et al. (2011) and Burr et al. (2013), but we introduce the broader social dilemma perspective into the analysis.

In the present paper, we identify and discuss various types of social dilemma in strategic R\&D behavior of enterprises. The analysis of firms' $R \& D$ behavior from the social dilemma perspective can be particularly useful for strategic managers and innovation policy makers. The first group can exploit the presented findings for the purposes of optimal decision making in the strategic contexts. The second group can use the discussed results to design a policy which resolves or overcomes identified social dilemmas.

The article proceeds as follows. The model of firms' behavior in R\&D is presented in the next section. The following section shows the firms' strategic games occurring under R\&D competition and R\&D coopetition. The last section presents and discusses obtained results, and in particular elaborates upon social dilemmas identified in strategic behavior of enterprises.

\section{Model}

We consider two scenarios of firms' R\&D strategic behavior. In both scenarios, firms choose whether to invest in an R\&D process, then they decide on the size of R\&D investments, and ultimately compete in a final product market through quantities produced. Both scenarios are modeled with three-stage games. This sequential approach follows the relevant literature, and in particular the seminal papers by d'Aspremont and Jacquemin (1988) and Kamien et al. (1992) as well as their 
excellent extensions by Amir et al. (2011) and Burr et al. (2013). We add one (our first) stage to the standard R\&D investment game, when firms can decide to bear an R\&D fixed entry cost or resign from investing in process innovation. The subsequent stages are similar to those considered in the cited literature, i.e., in the second stage firms determine the investment levels, and the last stage is devoted to output setting. The R\&D investments made by firms reduce the costs of production (hence, we talk about process innovation). The R\&D investments of all firms affect the individual firm cost function (in this sense, knowledge spillovers occur).

We stress that differently from the models mentioned in the introduction, in our model, there is an entry cost. This cost is interpreted as a cost of fixed assets, including, e.g., land, buildings, and infrastructure. These costs are further referred to as initial investment. In the second stage of a game, firms decide on sizes of R\&D investments which are interpreted as a cost of current assets and intangible assets, including patents, and the skills or talents of a workforce. In short, those assets are crucial to carrying out the actual research. In the last stage of the game, firms compete in the final good market in a Cournot duopoly (firms set their production outputs).

There are two scenarios considered. The first scenario is the standard competitive scenario, in which firms decide on entering $R \& D$, sizes of $R \& D$ investments and production levels in a competitive way. In particular, the R\&D investment levels are set independently and simultaneously. This scenario is similar to the game proposed in Burr et al. (2013). In the second scenario (R\&D coopetition), firms may choose to enter a binding agreement in the R\&D stage of the game. If the contract is signed, costs of tangible and intangible assets and all innovations are shared. Under R\&D contract, the R\&D investment levels are set by firms according to the Kalai-Smorodinsky bargaining rules.

It is assumed that there are two firms, indexed by $i=1$ and $i=2$. Firms produce quantities $q_{i} \geq 0, i=1,2$. The inverse demand for the product is given as a linear price function $p(Q)=a-Q$, where $Q=q_{1}+q_{2}$ and $a \geq 0$ is a demand intercept. The entry cost is denoted by $b>0$, while R\&D investments are denoted by $x_{1} \geq 0$ and $x_{2} \geq 0$.

It is usual to model R\&D investments and spillovers through results of an R\&D. For example, in Kamien et al. (1992), Amir et al. (2011) or Burr et al. (2013), the marginal cost is modeled as $c_{i}-x_{i}-\beta x_{-i}$, where $x_{i}$ is cost reduction level decided by a firm and $\beta$ is a parameter controlling the degree of spillovers (for a discussion, see, e.g., Cohen and Levinthal (1990)). The initial marginal cost is denoted by $c_{i}$.

In the proposed model, in a scenario without binding agreements, the total cost is given as

$$
c_{i}\left(q_{i}, x_{i}, x_{-i}, l_{i}\right)=c \cdot K\left(x_{i}\right) \cdot\left(1-\beta\left(1-K\left(x_{-i}\right)\right)\right) \cdot q_{i}+x_{i}+b \cdot l_{i},
$$

where $-i$ denotes the other firm, and for simplicity it is assumed that $c_{i}=c, i=1,2$. A function $K$ models the influence of $\mathrm{R} \& \mathrm{D}$ investments on the marginal production cost and is discussed later in detail. The general idea is that $K$ takes an amount of $\mathrm{R} \& \mathrm{D}$ investments and returns a level of cost reduction. Parameter $\beta$ controls a level of spillovers, but differently from the previous literature, it works on innovations 
rather than investments. If $\beta=0$, there are no spillovers. If $\beta=1$, all innovations benefit all firms. The rest of total cost is related to investments, where $l_{i}=0$ means that a firm decides to not invest in fixed assets and $l_{i}=1$ means the opposite. As mentioned before, $x_{i} \geq 0, i=1,2$ denote costs of intangible assets. It is assumed that $l_{i}=0$ implies $x_{i}=0$, but it is possible to have $l_{i}=1$ and $x_{i}=0$, that is, a firm may invest in a laboratory, but decide to not carry out any research.

In a scenario with a possibility to enter a binding agreement related to $R \& D$, the total cost differs significantly. In this case all investments are shared (knowledge sharing between cooperating partners occurs), and so are innovations. This situation is only possible when both firms decide to engage in an R\&D and sign a contract. In this situation total cost reads

$$
c_{i}\left(q_{i}, x_{1}, x_{2}, 1\right)=c \cdot K\left(x_{1}+x_{2}\right) \cdot q_{i}+x_{i}+\frac{b}{2} .
$$

The cost of fixed assets is shared equally, because we assume that firms are symmetric. Since R\&D investments are now decided within a contract, a bargaining problem is used. As is common, we employ the Kalai-Smorodinsky bargaining solution to determine $x_{i}, i=1,2$.

We assume that the function $K$ is of the following form

$$
K(x)= \begin{cases}e^{-\lambda x} & \text { for } x \geq 0 \\ 0 & \text { for } x<0\end{cases}
$$

The basic interpretation of this function is such that with no investments $x=0$ there is no cost reduction, because $K(0)=1$. The function decreases asymptotically to 0 , that is, cost reduction increases to $100 \%$ as investments increase to infinity.

To simplify the analysis, a symmetric model is assumed from the start. On top of that it is also assumed that $a>0, c>0$ and $\lambda>0$. Two additional properties are assumed.

Assumption 1 We assume that it is profitable for firms to produce positive amount of good regardless of the R\&D investments. Mathematically, we assume that $a>\psi \cdot c$ for $\psi>0$ large enough.

Assumption 2 We assume that the innovation process is efficient enough so that it profitable for firms to have positive R\&D investments, even without the possibility of entering a binding contract, given that $b=0$. Mathematically, we assume that $\lambda$ is large enough.

Assumption 1 guarantees existence of an equilibrium on a final product market with positive levels of production regardless of the amount of $R \& D$ investments. The technical meaning of this assumption becomes clear when examining first order optimality conditions for quantities produced at an equilibrium on the final product market. 
Assumption 2 is a condition regarding efficiency of R\&D process. For higher values of $\lambda$, cost reductions are higher given the same level of $R \& D$ investments $x_{i}, i=1,2$.

In what follows, it is assumed that there are no spillovers in competitive case, that is $\beta=0$. Thus, the relevant cost functions read

$$
c_{i}\left(q_{i}, x_{i}, l_{i}\right)=c \cdot K\left(x_{i}\right) \cdot q_{i}+x_{i}+b \cdot l_{i}
$$

outside of a contract and

$$
c_{i}\left(q_{i}, x_{1}, x_{2}, 1\right)=c \cdot K\left(x_{1}+x_{2}\right) \cdot q_{i}+x_{i}+\frac{b}{2}
$$

within a contract (spillovers via knowledge sharing occur). Profits are given, with a slight abuse of notation, as

$$
\pi_{i}\left(q_{1}, q_{2}, x_{1}, x_{2}\right)=\left(a-\left(q_{1}+q_{2}\right)\right) \cdot q_{i}-c_{i}\left(q_{i}, x_{i}, x_{-i}, l_{i}\right)
$$

for $i=1,2$.

\section{Firms' strategic games}

To efficiently analyze the game defined above, it is transformed into a strategic form game with only two strategies $l_{i} \in\{0,1\}$ for each firm. The payoffs in that game are derived through solving for best strategies in the next two stages of the threestage game using backward induction. This is done for all possible profiles of initial investments $\left(l_{1}, l_{2}\right)$ in a series of propositions.

The easiest case to analyze is where both firm decide to not make initial investments, that is, a profile $\left(l_{1}, l_{2}\right)=(0,0)$ is selected. The following proposition gives payoffs of firms for this profile of strategies.

Proposition 1 Given assumption 1, when both firms withdraw from initial $R \& D$ investments, that is $l_{i}=0, i=1,2$, firms' payoffs read

$$
\pi_{1}(0,0)=\frac{1}{9}(a-c)^{2}=\pi_{2}(0,0)
$$

These payoffs result from a unique and positive equilibrium.

The more delicate issue concerns the case where one firm withdraws from initial investment, but the other does not, that is, we deal with a profile $\left(l_{1}, l_{2}\right)=(1,0)$ or $\left(l_{1}, l_{2}\right)=(0,1)$. The following proposition gives firms' payoffs in these cases.

Proposition 2 Given assumptions 1 and 2, when one firm withdraws from initial investment, while the other does not, that is $l_{i}=0$ and $l_{-i}=1, i=1,2$, firms' payoffs read 


$$
\begin{aligned}
& \pi_{1}(1,0)=\frac{\lambda\left((a+c)^{2}-18 b\right)-18 \log \left(\frac{2}{9} c(\lambda(a+c)+\gamma)\right)+\gamma(a+c)-9}{18 \lambda} \\
& \pi_{2}(1,0)=\frac{(2(a-2 c)(\lambda(a+c)+\gamma)+9)^{2}}{36(\lambda(a+c)+\gamma)^{2}},
\end{aligned}
$$

where $\gamma=\sqrt{\lambda\left(\lambda(a+c)^{2}-18\right)}$. Payoffs at the profile $\left(l_{1}, l_{2}\right)=(0,1)$ are symmetric. In both cases, payoffs result from unique and positive equilibria at the final product market and positive $R \& D$ investments.

The last case is concerned with the profile $\left(l_{1}, l_{2}\right)=(1,1)$. The following proposition gives firms' payoffs in this case.

Proposition 3 Given assumptions 1 and 2, when both firms make initial investments, that is when $\left(l_{1}, l_{2}\right)=(1,1)$, firms' payoffs read

$$
\pi_{1}(1,1)=\frac{-36 \log \left(-\frac{2 c}{\sqrt{a^{2}-\frac{9}{\lambda}}-a}\right)+2 a^{2} \lambda+2 a \sqrt{\lambda\left(a^{2} \lambda-9\right)}-36 b \lambda-9}{36 \lambda}=\pi_{2}(1,1) .
$$

The above payoffs result from unique and positive equilibria at the final product market and positive $R \& D$ investments.

Summarizing, the strategic game that firms face is given as the following game $G$

\begin{tabular}{lll}
\hline & 0 & 1 \\
\hline 0 & $\left(\pi_{1}(0,0), \pi_{2}(0,0)\right)$ & $\left(\pi_{1}(0,1), \pi_{2}(0,1)\right)$ \\
1 & $\left(\pi_{1}(1,0), \pi_{2}(1,0)\right)$ & $\left(\pi_{1}(1,1), \pi_{2}(1,1)\right)$ \\
\hline
\end{tabular}

where $\pi_{i}(0,0), \pi_{i}(1,0), \pi_{i}(0,1)$ and $\pi_{i}(1,1)$ are given by formulas (1)-(3). Game $G$ is symmetric and so we can only deal with a payoff matrix (with a certain abuse of notation)

$$
G=\left(\begin{array}{ll}
\pi_{1}(0,0) & \pi_{1}(0,1) \\
\pi_{1}(1,0) & \pi_{1}(1,1)
\end{array}\right)
$$

As mentioned above, there is a possibility to introduce an institution of a binding R\&D agreement. Firms may sign such a contract only when they decide to invest in $\mathrm{R} \& \mathrm{D}$, that is when $\left(l_{1}, l_{2}\right)=(1,1)$. The following proposition gives firms' payoffs when they decide to sign a contract.

Proposition 4 Given assumptions 1 and 2, when both firms enter a binding $R \& D$ agreement, firms' payoffs read 


$$
\begin{aligned}
& \pi_{1}^{\mathrm{c}}=-\frac{\log \left(\frac{2}{9} c \sqrt{a^{2} \lambda^{2}-9 \lambda}+\frac{2 a c \lambda}{9}\right)}{2 \lambda}+\frac{a \sqrt{a^{2} \lambda^{2}-9 \lambda}}{18 \lambda}+\frac{a^{2}}{18}-\frac{b}{2}-\frac{1}{4 \lambda}, \\
& \pi_{2}^{\mathrm{c}}=-\frac{\log \left(\frac{2}{9} c \sqrt{a^{2} \lambda^{2}-9 \lambda}+\frac{2 a c \lambda}{9}\right)}{2 \lambda}+\frac{a \sqrt{a^{2} \lambda^{2}-9 \lambda}}{18 \lambda}+\frac{a^{2}}{18}-\frac{b}{2}-\frac{1}{4 \lambda} .
\end{aligned}
$$

The above payoffs stem from unique and positive equilibrium at the final product market and positive investments within an $R \& D$ agreement.

Introduction of a possibility to enter a binding $R \& D$ contract changes the previous game into the following game $G^{\mathrm{c}}$

\begin{tabular}{lll}
\hline & 0 & 1 \\
\hline 0 & $\left(\pi_{1}(0,0), \pi_{2}(0,0)\right)$ & $\left(\pi_{1}(0,1), \pi_{2}(0,1)\right)$ \\
1 & $\left(\pi_{1}(1,0), \pi_{2}(1,0)\right)$ & $\left(\pi_{1}^{\mathrm{c}}(1,1), \pi_{2}^{\mathrm{c}}(1,1)\right)$ \\
\hline
\end{tabular}

where $\pi_{i}^{\mathrm{c}}(1,1), i=1,2$ are given by (4). As previously, this game is symmetric and so we can only deal with a single payoff matrix (with a certain abuse of notation)

$$
G^{\mathrm{c}}=\left(\begin{array}{ll}
\pi_{1}(0,0) & \pi_{1}(0,1) \\
\pi_{1}(1,0) & \pi_{1}^{\mathrm{c}}(1,1)
\end{array}\right) .
$$

These two strategic games give a complete description of the strategic choices of both firms in two scenarios, the first without a possibility of entering a binding R\&D contract, and the second with such a possibility.

To simplify further discussion, we normalize the initial marginal cost $c=1$. Thus, values of all other parameters are given in terms of this initial marginal cost. The normalization leaves only three exogenous parameters $a, \lambda$ and $b$ and all further discussion is kept in terms of those three parameters.

The assumptions 1 and 2 guarantee that for appropriately large values of exogenous parameters, equilibrium production levels and $R \& D$ investments are positive. It is useful for further discussion to derive precise bounds for these parameters. For example, optimizing in a profile $\left(l_{1}, l_{2}\right)=(0,0)$ for $q_{i}$ leads to the following optimal production levels $q_{i}=(a-c) / 3$. In order to keep optimal production levels positive, it is necessary to assume that $a>c$. In the same vein, when $\left(l_{1}, l_{2}\right)=(1,0)$, the same optimization leads to optimal production level $q_{2}=\left(a-c\left(2-e^{-\lambda x_{1}}\right)\right) / 3$. It is necessary to assume that $a>2 c$ to keep the optimal production level positive for all values of R\&D investments $x_{1}>0$. Continuing in this manner, the necessary conditions on the exogenous parameters, and taking into account the normalization $c=1$, are

$$
a>2 \quad \text { and } \quad \lambda>\frac{9}{4(a-1)}
$$

Figure 1 shows the set of all viable values of exogenous parameters. 


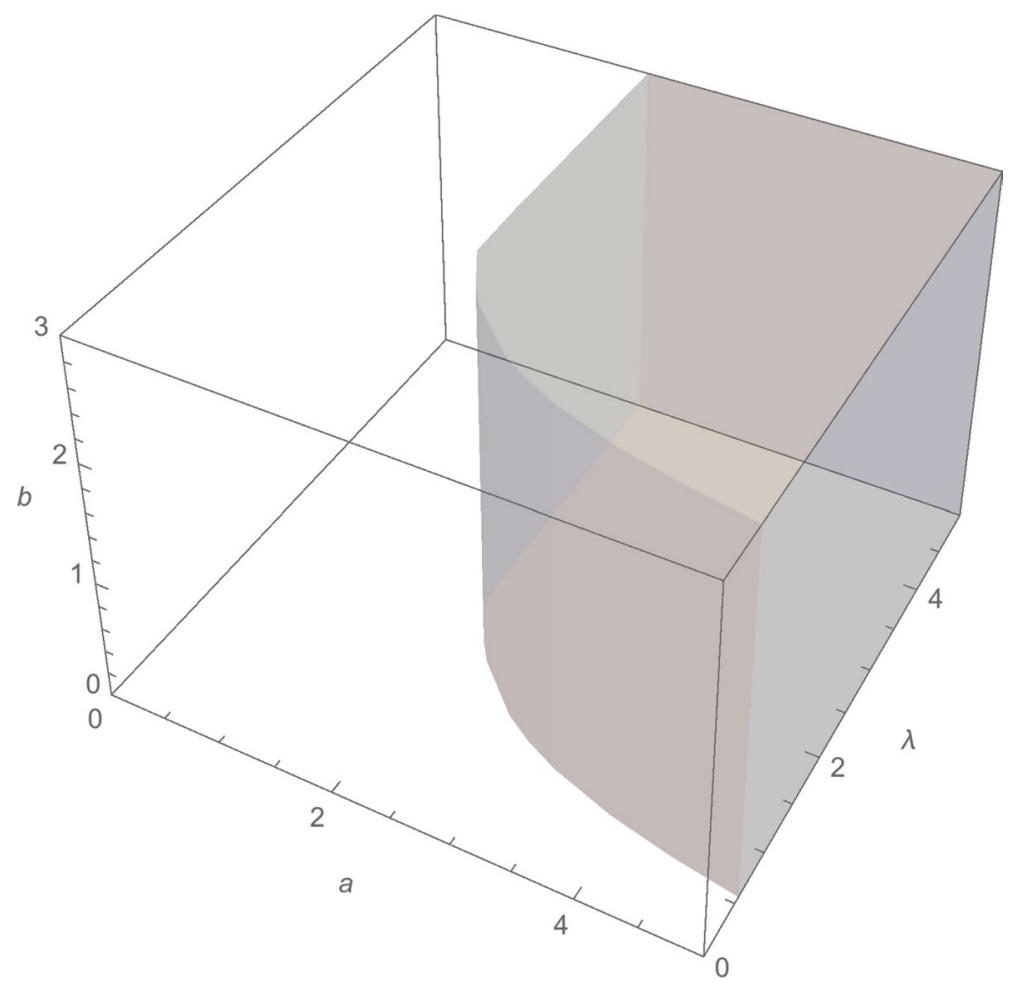

Fig. 1 Set of viable values of exogenous parameters $a, \lambda$ and $b$

Depending on a particular values of parameters $a, \lambda$ and $b$, there are different situations that can be encountered. To simplify the discussion we have the following proposition.

Proposition 5 Let values of the parameters a, $\lambda$ satisfy conditions (5) and letc $=1$. Then the following inequalities

$$
\pi_{1}(0,0)>\pi_{1}(0,1), \quad \pi_{1}(1,0)>\pi_{1}(1,1), \quad \pi_{1}(1,1)<\pi_{1}^{\mathrm{c}}(1,1)
$$

are satisfied. Symmetric inequalities for $i=2$ are also satisfied.

The above proposition allows dividing the set of valid values of parameters into separate regions, each characterized by a different behavior.

\section{Results and discussion}

Proposition 5 simplifies the discussion on strategic behavior of firms. If there is no possibility to enter a binding agreement, there are only two inequalities that can change, depending on values of exogenous parameters. In particular, the whole set 


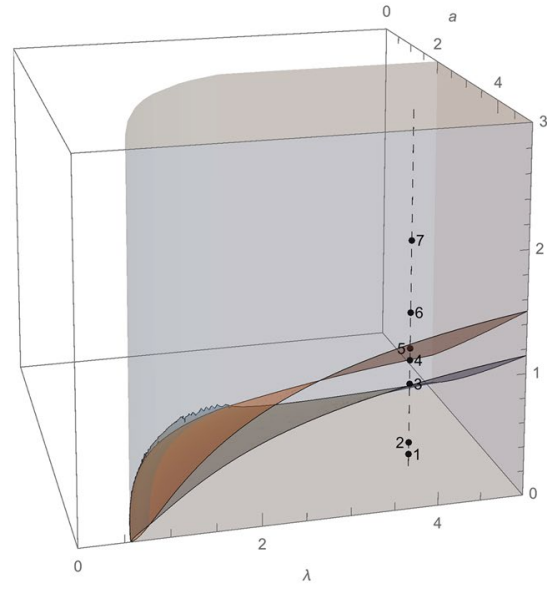

(a) without a contract

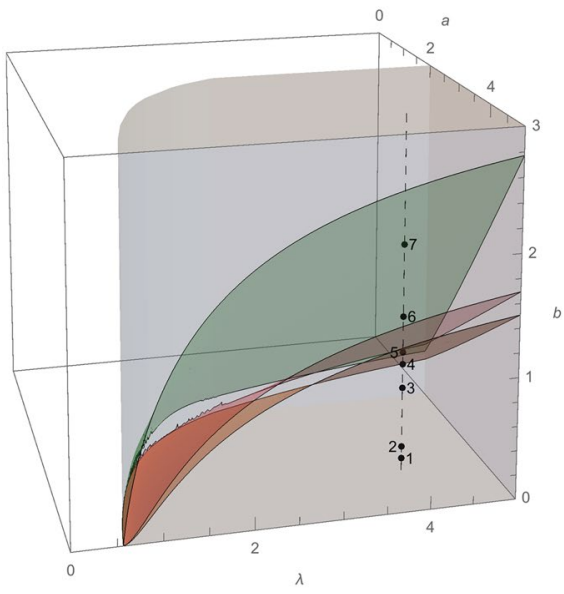

(b) with a contract

Fig. 2 A set of valid values of exogenous parameters divided into regions based on inequalities between firms' payoffs

of valid values of parameters is divided into separate regions by two surfaces defined by $\pi_{1}(0,0)=\pi_{1}(1,0)$ and $\pi_{1}(0,1)=\pi_{1}(1,1)$. Figure $2 \mathrm{a}$ shows the set of valid values of parameters divided with the two surfaces.

When there is a possibility to sign a contract, there are three possible surfaces defined by $\pi_{1}(0,0)=\pi_{1}(1,0), \pi_{1}(0,1)=\pi_{1}^{\mathrm{c}}(1,1)$ and $\pi_{1}(1,0)=\pi_{1}^{\mathrm{c}}(1,1)$. Figure $2 \mathrm{~b}$ shows the set of valid values of parameters divided with the three surfaces. Figures $2 \mathrm{a}$ and $2 \mathrm{~b}$ contain also a line for $a=4, \lambda=4$ and $b>0$ with seven points corresponding to seven values of $b$. This points constitute seven examples considered further in this section.

It may seem that, for example, in the case of a game without a contract, there are only three separate situations, and indeed, there are only three types of games as far as Nash equilibrium is concerned. However, we can have a coordination game with one or the other Nash equilibrium being a risk dominant equilibrium, and because of that fact we need seven examples to show the variety of possible strategic situations. All considered examples have $a=4$ and $\lambda=4$, while the initial R\&D cost $b$ is varied from the very low (example 1) to the relatively high (example 7).

Example 1 Let $b=1 / 10$, that is the initial $\mathrm{R} \& \mathrm{D}$ cost is small. In this case, the game without a contract is a harmony game and reads

\begin{tabular}{lll}
\hline & 0 & 1 \\
\hline 0 & $(1.00,1.00)$ & $(0.50,1.89)$ \\
1 & $(1.89,0.50)$ & $(1.07,1.07)$ \\
\hline
\end{tabular}


If the initial $R \& D$ cost is low, there is only one Nash equilibrium at which both firms make R\&D investments. The equilibrium profile is also Pareto optimal. In this case introduction of a contract gives the following game

\begin{tabular}{lll}
\hline & 0 & 1 \\
\hline 0 & $(1.00,1.00)$ & $(0.50,1.89)$ \\
1 & $(1.89,0.50)$ & $(1.36,1.36)$ \\
\hline
\end{tabular}

As we may observe, a scenario with a possibility to sign a contract has the same Nash equilibrium, and the equilibrium is also Pareto optimal. Concluding, for a low initial R\&D cost, both firms engage in R\&D. Also, since the equilibrium is Pareto there are no incentives to change this behavior. It seems that this case is the most desirable one.

Example 2 Let $b=2 / 10$. In this case, the game without a contract reads

\begin{tabular}{lll}
\hline & 0 & 1 \\
\hline 0 & $(1.00,1.00)$ & $(0.50,1.79)$ \\
1 & $(1.79,0.50)$ & $(0.97,0.97)$ \\
\hline
\end{tabular}

Observe that with the rising initial R\&D cost a strategic situation changes. The profile invest-invest is still the unique Nash equilibrium, yet the game is now a prisoner's dilemma, where the Nash equilibrium is not Pareto optimal. This case has been already presented in Amir et al. (2011) and Burr et al. (2013). According to those authors, the prisoner's dilemma in R\&D implies that, when spillovers are small, as is assumed throughout the current text, firms find it to their advantage to engage in untypical collusion: jointly refraining from engaging in R\&D, a phenomenon called an R\&D-avoiding cartel.

Introduction of a contract gives the following game

\begin{tabular}{lll}
\hline & 0 & 1 \\
\hline 0 & $(1.00,1.00)$ & $(0.50,1.79)$ \\
1 & $(1.79,0.50)$ & $(1.31,1.31)$ \\
\hline
\end{tabular}

In this case, an introduction of a binding $R \& D$ agreement changes the Nash equilibrium into the Pareto optimal profile, thus removing incentives to form an R\&D-avoiding cartel, as discussed in Amir et al. (2011).

Example 3 Let $b=7 / 10$. In this case, the game without a contract reads 


\begin{tabular}{lll}
\hline & 0 & 1 \\
\hline 0 & $(1.00,1.00)$ & $(0.50,1.29)$ \\
1 & $(1.29,0.50)$ & $(0.47,0.47)$ \\
\hline
\end{tabular}

Observe that with a higher initial $R \& D$ cost, a strategic situation changes yet again, and we obtain a chicken game. This time, there are two asymmetric Nash equilibria, at which one firm invests, and the other does not. With a medium initial $R \& D$ cost and a competitive fight within an investment market, there is not enough space for both firms.

This situation leads to a massively unbalanced market including highly technologically advanced firms and firms falling behind. In the long run, we can expect a monopoly either through a takeover or a firm dropping out of a market. Note that entry $R \& D$ cost plays here a role of deterrence tool. One firm discourages the other from investing in $R \& D$. In the literature, deterrence strategy is usually discussed in the context of industry entry, but here we have a specific case of R\&D investment market deterrence. In particular, Dasgupta and Stiglitz (1980) and Reinganum (1983) show that incumbents can effectively discourage possible entrants by introducing innovative products or cost-reducing technologies (process innovations).

Introduction of a contract in this particular case gives the following game

\begin{tabular}{lll}
\hline & 0 & 1 \\
\hline 0 & $(1.00,1.00)$ & $(0.50,1.29)$ \\
1 & $(1.29,0.50)$ & $(1.06,1.06)$ \\
\hline
\end{tabular}

The new game has only one Nash equilibrium: invest-invest, which is Pareto optimal. Thus, an introduction of an R\&D contract changes a strategic situation to the balanced one and from this point of view is more desirable than the asymmetric outcome.

Example 4 Let $b=9 / 10$. In this case, the game without a contract reads

\begin{tabular}{lll}
\hline & 0 & 1 \\
\hline 0 & $(1.00,1.00)$ & $(0.50,1.09)$ \\
1 & $(1.09,0.50)$ & $(0.27,0.27)$ \\
\hline
\end{tabular}

Without a contract, there is still just enough room for only a single firm investing in R\&D. This situation is not desirable as is discussed in an example 3.

Introduction of a contract gives the following game

\begin{tabular}{lll}
\hline & 0 & 1 \\
\hline 0 & $(1.00,1.00)$ & $(0.50,1.09)$ \\
\hline
\end{tabular}




\begin{tabular}{lll}
\hline & 0 & 1 \\
\hline 1 & $(1.09,0.50)$ & $(0.96,0.96)$ \\
\hline
\end{tabular}

With a contract, investing is a dominant strategy. However, with a higher cost, the only Nash equilibrium invest-invest fails to be a Pareto optimal profile. The game is a prisoner's dilemma and the main motive behind $R \& D$ investments is a mix of fear and greed. There are clear incentives to form an R\&D-avoiding cartel as discussed in Amir et al. (2011). However, it can be argued that with a proper discriminating antitrust policy, this situation is more desirable than the asymmetric outcome.

Example 5 Let $b=1$. In this case, the game without a contract reads

\begin{tabular}{lll}
\hline & 0 & 1 \\
\hline 0 & $(1.00,1.00)$ & $(0.50,0.99)$ \\
1 & $(0.99,0.50)$ & $(0.17,0.17)$ \\
\hline
\end{tabular}

The unique Nash equilibrium is a profile not invest-not invest, which is Pareto optimal. Introduction of a contract changes a game to the following

\begin{tabular}{lll}
\hline & 0 & 1 \\
\hline 0 & $(1.00,1.00)$ & $(0.50,0.99)$ \\
1 & $(0.99,0.50)$ & $(0.91,0.91)$ \\
\hline
\end{tabular}

This time investing is not a dominant strategy. There are two equilibria, first without investments and the other with investments. The game is a trust dilemma (also called an assurance game).

Example 6 Let $b=13 / 10$. In this case, the game without a contract reads

\begin{tabular}{lll}
\hline & 0 & 1 \\
\hline 0 & $(1.00,1.00)$ & $(0.50,0.69)$ \\
1 & $(0.69,0.50)$ & $(-0.13,-0.13)$ \\
\hline
\end{tabular}

The unique Nash equilibrium is still a profile not invest-not invest, which is Pareto optimal. Introduction of a contract changes a game to the following

\begin{tabular}{lll}
\hline & 0 & 1 \\
\hline 0 & $(1.00,1.00)$ & $(0.50,0.69)$ \\
1 & $(0.69,0.50)$ & $(0.76,0.76)$ \\
\hline
\end{tabular}


There are still two equilibria, the first without investments and the other with investments. However, for such a high cost of initial R\&D investment, the equilibrium without investments becomes a risk dominant equilibrium.

Example 7 Let $b=19 / 10$. In this case, the game without a contract reads

\begin{tabular}{lll}
\hline & 0 & 1 \\
\hline 0 & $(1.00,1.00)$ & $(0.50,0.09)$ \\
1 & $(0.09,0.50)$ & $(-0.73,-0.73)$ \\
\hline
\end{tabular}

As before, the unique Nash equilibrium is still a profile not invest-not invest, which is Pareto optimal. Introduction of a contract changes a game to the following

\begin{tabular}{lll}
\hline & 0 & 1 \\
\hline 0 & $(1.00,1.00)$ & $(0.50,0.09)$ \\
1 & $(0.09,0.50)$ & $(0.46,0.46)$ \\
\hline
\end{tabular}

Observe that for the high enough initial cost $b$, even an introduction of a contract, that rises firms' profits while investing in $R \& D$, cannot incentivize firms to do so.

The above examples illustrate typical strategic situations related to R\&D investments with and without a possibility to enter a binding R\&D contract. Only one strategic dilemma, the prisoner's dilemma, has been previously reported in the literature. We show, that in fact, all typical social dilemmas, comprising fear, greed and a mix of fear and greed, are present in R\&D games, depending on an initial R\&D cost $b$, a marginal cost $c$, a size of a market $a$, efficiency of an R\&D process $\lambda$ and possibility to sign an R\&D contract. Moreover, we show that in each of the distinct cases, a possibility to enter a binding $R \& D$ agreement, that is to engage in a bargaining process concerned with sharing $\mathrm{R} \& \mathrm{D}$ costs, changes a situation to a more desirable one, either through changing a Nash equilibrium to a Pareto optimal equilibrium or by introducing invest-invest Nash equilibrium. The only exception is the case of a very large initial $\mathrm{R} \& \mathrm{D}$ cost $b$.

\section{Conclusions}

This paper shows that social dilemmas associated with the firms' competitive behavior are mitigated by $R \& D$ coopetition. It is worth stressing that $R \& D$ agreements can: (i) effectively eliminate firms' incentives to form an R\&D avoiding cartel when the initial $R \& D$ cost is not too high (example 2), (ii) prevent the monopolization of the industry (examples 3 and 4) or (iii) induce R\&D investments which can lead to innovations (examples 5 and 6). Such implications are interesting to innovation and competition policymakers and managers since, as we demonstrate, R\&D agreements 
have the potential to stimulate innovation in the industry and, at the same time, prevent cartelization or monopolization.

Clearly, the above conclusions depend on the introduced assumptions and the presence of the R\&D entry cost in our model. However, such a cost is not rare in business practice, and the value of such cost is quite differentiated in real-world situations, as in our model. The cost $b$ in our model is given exogenously and irrespective of all other parameters, particularly the size of the market and R\&D efficiency. Thus, when we talk about the high initial investments, they are high to potential profits since all other parameters determining profits are kept constant.

The paper elaborates on an institution of an $R \& D$ contract regarding social dilemmas that occur commonly in such cases. As such, it is interesting from the policy-making perspective. However, even if that contract type is allowed, a decision to engage in $R \& D$ coopetition is down to managers. From this point of view, it is imperative that high-level managers making such decisions understand the decisions' ramifications and implications.

Lastly, the present study can be extended in numerous ways. For example, one can think of introducing uncertainty into the investment process. Another idea is to consider an asymmetric game in which one enterprise plans to enter the industry but has to bear an entry cost. In contrast, the other enterprise (the incumbent) has already invested in fixed assets. Also, absorptive capacity can be introduced into the model to differentiate between firms' abilities.

\section{Proofs}

The appendix contains proofs of all propositions.

Proof of proposition 1 This case constitutes a standard Cournot duopoly and is presented here for convenience. General profits of firms when $l_{i}=0, i=1,2 \mathrm{read}$

$$
\begin{aligned}
& \pi_{1}=q_{1}\left(a-q_{1}-q_{2}\right)-c q_{1}, \\
& \pi_{2}=q_{2}\left(a-q_{1}-q_{2}\right)-c q_{2} .
\end{aligned}
$$

Omitting standard computations, the unique equilibrium in the final good market reads

$$
q_{1}=\frac{a-c}{3}, \quad q_{2}=\frac{a-c}{3} .
$$

Due to an assumption 1, this equilibrium is positive, in the sense that optimal production levels are positive. Substituting the above optimal production outputs into the general profit functions yields payoffs (1).

Proof of proposition 2 Only case $\left(l_{1}, l_{2}\right)=(1,0)$ is considered since the other profile leads to a symmetric situation. When a profile $\left(l_{1}, l_{2}\right)=(1,0)$ is played, firms' general profits read 


$$
\begin{aligned}
& \pi_{1}=q_{1}\left(a-c e^{-\lambda x_{1}}-q_{2}\right)-b-q_{1}^{2}-x_{1}, \\
& \pi_{2}=q_{2}\left(a-q_{1}-q_{2}\right)-c q_{2} .
\end{aligned}
$$

Standard computations lead to the unique equilibrium on the final good market

$$
\begin{aligned}
& q_{1}=\frac{1}{3}\left(a-2 c e^{-\lambda x_{1}}+c\right), \\
& q_{2}=\frac{1}{3}\left(a+c\left(e^{-\lambda x_{1}}-2\right)\right) .
\end{aligned}
$$

Due to assumption 1 we may assume that $a>2 c$ and consequently for any $x_{1} \geq 0$ the above optimal productions are always positive.

The profit of the first firm at the above equilibrium reads

$$
\pi_{1}=\frac{1}{9}\left(e^{-2 \lambda x_{1}}\left((a+c) e^{\lambda x_{1}}-2 c\right)^{2}-9 b-9 x_{1}\right) .
$$

The above expression is a quadratic function in $e^{\lambda x_{1}}$ and can be easily maximized leading to the optimal investment

$$
x_{1}=\frac{\log \left(\frac{2}{9} c\left(\lambda(a+c)+\sqrt{\lambda\left(\lambda(a+c)^{2}-18\right)}\right)\right)}{\lambda} .
$$

Due to assumption 2 for $\lambda>0$ large enough expression $\lambda\left(\lambda(a+c)^{2}-18\right)$ is arbitrarily large and the whole argument of a log function is above 1 , hence, the optimal R\&D investment is positive. Substituting the above optimal investment into the profit functions yields the optimal profits (2).

Proof of proposition 3 For $\left(l_{1}, l_{2}\right)=(1,1)$ firms' profits read

$$
\begin{aligned}
& \pi_{1}=q_{1}\left(a-c e^{-\lambda x_{1}}-q_{2}\right)-b-q_{1}^{2}-x_{1}, \\
& \pi_{2}=q_{2}\left(a-c e^{-\lambda x_{2}}-q_{1}\right)-b-q_{2}^{2}-x_{2} .
\end{aligned}
$$

Optimization with respect to quantities leads to the following equilibrium at the final good market

$$
\begin{aligned}
& q_{1}=\frac{1}{3}\left(a+c\left(e^{-\lambda x_{2}}-2 e^{-\lambda x_{1}}\right)\right), \\
& q_{2}=\frac{1}{3}\left(a+c\left(e^{-\lambda x_{1}}-2 e^{-\lambda x_{2}}\right)\right) .
\end{aligned}
$$

Due to assumption 1, we may assume that $a>2 c$ and consequently, for any $x_{i} \geq 0$, optimal production levels are positive.

Substituting the above optimal production outputs into the firms' general profits yields the following formulas 


$$
\begin{aligned}
& \pi_{1}=\frac{1}{9}\left(e^{-2 \lambda\left(x_{1}+x_{2}\right)}\left(a e^{\lambda\left(x_{1}+x_{2}\right)}+c\left(e^{\lambda x_{1}}-2 e^{\lambda x_{2}}\right)\right)^{2}-9 b-9 x_{1}\right), \\
& \pi_{2}=\frac{1}{9}\left(e^{-2 \lambda\left(x_{1}+x_{2}\right)}\left(a e^{\lambda\left(x_{1}+x_{2}\right)}+c\left(e^{\lambda x_{2}}-2 e^{\lambda x_{1}}\right)\right)^{2}-9 b-9 x_{2}\right) .
\end{aligned}
$$

Differentiating the above expressions with respect to $x_{1}$ and $x_{2}$ respectively, and equating them to 0 , yields a system of equalities that are quadratic in $e^{\lambda x_{i}}$ and can be solved for the optimal level of R\&D investments that read

$$
x_{1}=\frac{\log \left(\frac{2 c}{a-\sqrt{a^{2}-\frac{9}{\lambda}}}\right)}{\lambda}=x_{2} .
$$

Due to an assumption 2 , for $\lambda>0$ large enough the expression $\sqrt{a^{2}-9 / \lambda}$ is positive and arbitrarily close to $a$ making the whole argument of a log function larger than 1 . Thus, optimal R\&D investments are positive. Substituting optimal production levels and optimal investments into firms' general profits gives optimal profits (3).

Proof of proposition 4 Firms' general profits within a binding R\&D contract read

$$
\begin{aligned}
& \pi_{1}=q_{1}\left(a-c e^{-\lambda\left(x_{1}+x_{2}\right)}-q_{1}-q_{2}\right)-\frac{b}{2}-x_{1}, \\
& \pi_{2}=q_{2}\left(a-c e^{-\lambda\left(x_{1}+x_{2}\right)}-q_{1}-q_{2}\right)-\frac{b}{2}-x_{2} .
\end{aligned}
$$

Optimal production is given as

$$
q_{1}=\frac{1}{3}\left(a-c e^{-\lambda\left(x_{1}+x_{2}\right)}\right), \quad q_{2}=\frac{1}{3}\left(a-c e^{-\lambda\left(x_{1}+x_{2}\right)}\right) .
$$

Due to an assumption 1 we may have $a>c$ and, consequently, optimal production levels are positive.

Substituting the above optimal production into the firms' profits yields the following formulas

$$
\begin{aligned}
& f_{1}\left(x_{1}, x_{2}\right)=\frac{1}{18}\left(2 a\left(a-2 c e^{-\lambda\left(x_{1}+x_{2}\right)}\right)-9 b+2 c^{2} e^{-2 \lambda\left(x_{1}+x_{2}\right)}-18 x_{1}\right) \\
& f_{2}\left(x_{1}, x_{2}\right)=\frac{1}{18}\left(2 a\left(a-2 c e^{-\lambda\left(x_{1}+x_{2}\right)}\right)-9 b+2 c^{2} e^{-2 \lambda\left(x_{1}+x_{2}\right)}-18 x_{2}\right),
\end{aligned}
$$

where we use $f_{i}$ to denote payoffs at the final market equilibrium. Optimal R\&D investments within a contract are calculated as the Kalai-Smorodinsky bargaining solution. Figure 3 shows a set of viable profit vectors $V=\left\{\left(f_{1}, f_{2}\right): x_{1} \geq 0, x_{2} \geq 0\right\}$.

To solve for the Kalai-Smorodinsky bargaining solution we need to find the Pareto optimal boundary of the set $V$. This boundary is composed of parts of curves $f_{1}\left(x_{1}, 0\right), x_{1} \geq 0$ and $f_{2}\left(0, x_{2}\right), x_{2} \geq 0$ and an envelope line that can be calculated as 


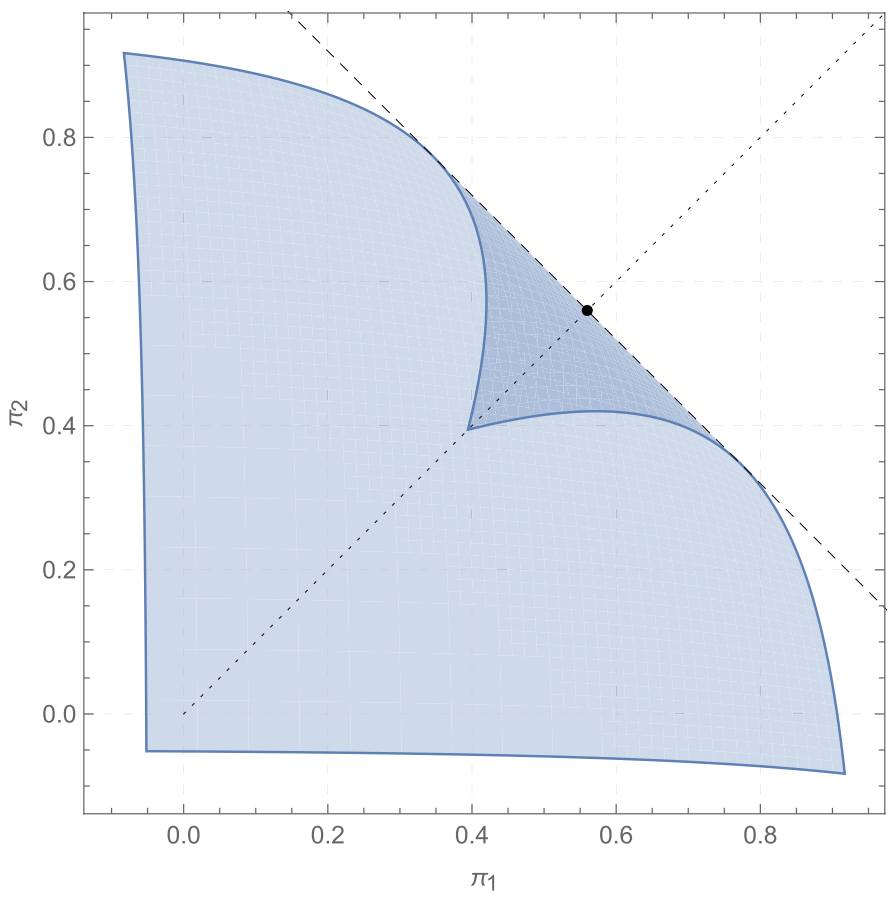

Fig. 3 Set of viable payoffs within a contract (blue). Numerical example for $a=3, b=1 / 10, c=1, \lambda=3$. A marked point is the Kalai-Smorodinsky bargaining solution. A dashed line is an envelop and constitutes the Pareto boundary

$$
\frac{\partial f_{1}}{\partial x_{1}} \frac{\partial f_{2}}{\partial x_{2}}-\frac{\partial f_{1}}{\partial x_{2}} \frac{\partial f_{2}}{\partial x_{1}}=0
$$

Simple algebra leads to the following line

$$
x_{1}+x_{2}=\frac{\log \left(\frac{2}{9} c \lambda\left(a+\sqrt{\left(a^{2}-\frac{9}{\lambda}\right)}\right)\right)}{\lambda}
$$

or in terms of profits to

$$
\pi_{1}+\pi_{2}=\frac{2 \lambda\left(a^{2}-9 b\right)-18 \log \left(\frac{2}{9} c\left(\sqrt{\lambda\left(a^{2} \lambda-9\right)}+a \lambda\right)\right)+2 a \sqrt{\lambda\left(a^{2} \lambda-9\right)}-9}{18 \lambda} .
$$

Since the solution is symmetric, the Kalai-Smorodinsky bargaining solution is given as (4). Uniqueness of optimal investments is obvious. The expression 


$$
\frac{\log \left(\frac{2}{9} c \lambda\left(a+\sqrt{\left(a^{2}-\frac{9}{\lambda}\right)}\right)\right)}{\lambda}
$$

is positive due to an assumption 2 what completes the proof.

Proof of proposition 5 We provide only a sketch of the proof because the proof involves only a tedious algebra.

The first inequality $\pi_{1}(0,0)>\pi_{1}(0,1)$ can be rewritten as

$$
\lambda\left(a\left(5 \sqrt{(a+1)^{2}-\frac{18}{\lambda}}-5 a+18\right)-7 \sqrt{(a+1)^{2}-\frac{18}{\lambda}}-17\right)+9>0
$$

that leads to the following conditions

$$
\left(a<3 \wedge(a+1)^{2} \lambda \geq 18\right) \vee(a \geq 3 \wedge 4(a-1) \lambda>9),
$$

but since for $2<a<3$ we have

$$
\frac{9}{4(a-1)}>\frac{18}{(a+1)^{2}}
$$

we can see that conditions (5) guarantee the postulated inequality.

The second inequality $\pi_{1}(1,0)>\pi_{1}(1,1)$ can be rewritten as

$$
\begin{aligned}
& 2 \lambda\left(a\left(-\sqrt{a^{2}-\frac{9}{\lambda}}+\sqrt{(a+1)^{2}-\frac{18}{\lambda}}+2\right)+\sqrt{(a+1)^{2}-\frac{18}{\lambda}}+1\right) \\
& -36 \log \left(a-\sqrt{a^{2}-\frac{9}{\lambda}}\right)-36 \log \left(\lambda\left(\sqrt{(a+1)^{2}-\frac{18}{\lambda}}+a+1\right)\right) \\
& -9+72 \log (3)>0
\end{aligned}
$$

First, substituting $\lambda=9 /(4(a-1))$ we see that the left hand side of the above expression equals 0 , that is, on a boundary the left hand side of the above expression is 0 . We now show that the derivative of the left hand side of the above inequality with respect to $\lambda$ is positive. This derivative equals

$$
\begin{aligned}
& a\left(-2 \sqrt{a^{2}-\frac{9}{\lambda}}+\frac{9}{\sqrt{\lambda\left(a^{2} \lambda-9\right)}}+2 \sqrt{(a+1)^{2}-\frac{18}{\lambda}}+4\right) \\
& +2\left(\sqrt{(a+1)^{2}-\frac{18}{\lambda}}+1\right) .
\end{aligned}
$$

The derivative is positive if and only if 


$$
\begin{aligned}
& \left(a \leq 1+\sqrt{2} \wedge a^{2} \lambda>9\right) \vee\left(1+\sqrt{2}<a<3+2 \sqrt{3} \wedge(a+1)^{2} \lambda \geq 18\right) \\
& \vee(a=3+2 \sqrt{3} \wedge 2 \lambda+36 \sqrt{3}>63) \\
& \quad \vee\left(a>3+2 \sqrt{3} \wedge \lambda>\frac{9(a+\sqrt{2 a+1}+1)}{2 a^{2} \sqrt{2 a+1}}\right),
\end{aligned}
$$

but for all intervals of $a$, if $\lambda$ satisfies condition (5) then it also satisfies the above conditions, hence the above derivative is positive and consequently the postulated inequality is satisfied.

Finally, the last inequality $\pi_{1}(1,1)<\pi_{1}^{\mathrm{c}}(1,1)$ can be rewritten as

$$
-2 \log \left(a-\sqrt{a^{2}-\frac{9}{\lambda}}\right)-\log \left(\lambda\left(\sqrt{a^{2}-\frac{9}{\lambda}}+a\right)\right)+b \lambda+\log (18)>0 .
$$

Substituting $\lambda=9 /(4(a-1))$ into the above expression yields

$$
\frac{9 b}{4(a-1)}
$$

which is a positive expression for any $b>0$ and $a>2$. The derivative of the left hand side of the above inequality with respect to $\lambda$ gives

$$
\frac{3 \sqrt{a^{2}-\frac{9}{\lambda}}+a}{2 \lambda \sqrt{a^{2}-\frac{9}{\lambda}}}+b-\frac{1}{\lambda} .
$$

This derivative is positive if and only if

$$
a^{2} \lambda>9 \wedge a \sqrt{\frac{\lambda}{a^{2} \lambda-9}}+2 b \lambda+1>0 .
$$

The first inequality is satisfied if $\lambda>9 /(4(a-1))$, and then obviously the other is true as well, what completes the proof.

Funding This research was supported by National Science Centre, Poland (Grant number 2016/21/B/ HS4/03016).

\section{Declarations}

Conflict of interest The authors declare that they have no competing interests. 
Open Access This article is licensed under a Creative Commons Attribution 4.0 International License, which permits use, sharing, adaptation, distribution and reproduction in any medium or format, as long as you give appropriate credit to the original author(s) and the source, provide a link to the Creative Commons licence, and indicate if changes were made. The images or other third party material in this article are included in the article's Creative Commons licence, unless indicated otherwise in a credit line to the material. If material is not included in the article's Creative Commons licence and your intended use is not permitted by statutory regulation or exceeds the permitted use, you will need to obtain permission directly from the copyright holder. To view a copy of this licence, visit http://creativecommons.org/licen ses/by/4.0/.

\section{References}

Amir, R., Garcia, F., Halmenschlager, C., \& Pais, J. (2011). R\&D as a prisoner's dilemma and R\&Davoiding cartels. The Manchester School, 79(1), 81-99.

Amir, R., Liu, H., Machowska, D., \& Resende, J. (2019). Spillovers, subsidies, and second-best socially optimal R\&D. Journal of Public Economic Theory, 21(6), 1200-1220.

Bouncken, R. B., \& Fredrich, V. (2016). Learning in coopetition: Alliance orientation, network size, and firm types. Journal of Business Research, 69(5), 1753-1758.

Bourreau, M., Doğan, P., \& Manant, M. (2016). Size of RJVs with partial cooperation in product development. International Journal of Industrial Organization, 46, 77-106.

Burr, C., Knauff, M., \& Stepanova, A. (2013). On the prisoner's dilemma in R\&D with input spillovers and incentives for R\&D cooperation. Mathematical Social Sciences, 66(3), 254-261.

Capuano, C., \& Grassi, I. (2019). Spillovers, product innovation and R\&D cooperation: A theoretical model. Economics of Innovation and New Technology, 28(2), 197-216.

Cohen, W. M., \& Levinthal, D. A. (1990). Absorptive capacity: A new perspective on learning and innovation. Administrative Science Quarterly, 35, 128-152.

Conti, C., \& Marini, M. A. (2019). Are you the right partner? R\&D agreement as a screening device. Economics of Innovation and New Technology, 28(3), 243-264.

Cosandier, C., Feo, G. D., \& Knauff, M. (2017). Equal treatment and socially optimal R\&D in duopoly with one-way spillovers. Journal of Public Economic Theory, 19(6), 1099-1117.

Cygler, J., Sroka, W., Solesvik, M., \& Dębkowska, K. (2018). Benefits and drawbacks of coopetition: The roles of scope and durability in coopetitive relationships. Sustainability, 10(8), 2688.

Dasgupta, P., \& Stiglitz, J. (1980). Industrial structure and the nature of innovative activity. The Economic Journal, 90(358), 266-293.

d'Aspremont, C., \& Jacquemin, A. (1988). Cooperative and noncooperative R\&D in duopoly with spillovers. The American Economic Review, 78(5), 1133-1137.

Jakobsen, S. (2020). Managing tension in coopetition through mutual dependence and asymmetries: A longitudinal study of a Norwegian R\&D alliance. Industrial Marketing Management, 84, 251-260.

Kamien, M. I., Muller, E. \& Zang, I. (1992). Research joint ventures and R\&D cartels. The American Economic Review, 82(5), 1293-1306.

Kamien, M. I., \& Zang, I. (2000). Meet me halfway: Research joint ventures and absorptive capacity. International Journal of Industrial Organization, 18(7), 995-1012.

Katz, M. L. (1986). An analysis of cooperative research and development. The RAND Journal of Economics, 17(4), 527-543. https://doi.org/10.2307/2555479

Kiyonari, T., Tanida, S., \& Yamagishi, T. (2000). Social exchange and reciprocity: Confusion or a heuristic? Evolution and Human Behavior, 21(6), 411-427.

Lambertini, L., \& Rossini, G. (1998). Product homogeneity as a prisoner's dilemma in a duopoly with R\&D. Economics Letters, 58(3), 297-301.

Płatkowski, T. (2017). Greed and fear in multiperson social dilemmas. Applied Mathematics and Computation, 308, 157-160.

Reinganum, J. F. (1983). Uncertain innovation and the persistence of monopoly. The American Economic Review, 73(4), 741-748.

Ritala, P., \& Sainio, L.-M. (2014). Coopetition for radical innovation: Technology, market and businessmodel perspectives. Technology Analysis \& Strategic Management, 26(2), 155-169.

Schumpeter, J. A. (1942). Socialism, capitalism and democracy. Harper and Brothers. 
Spence, M. (1984). Cost reduction, competition and industry performance. Econometrica, 52, 101-121.

Publisher's Note Springer Nature remains neutral with regard to jurisdictional claims in published maps and institutional affiliations. 